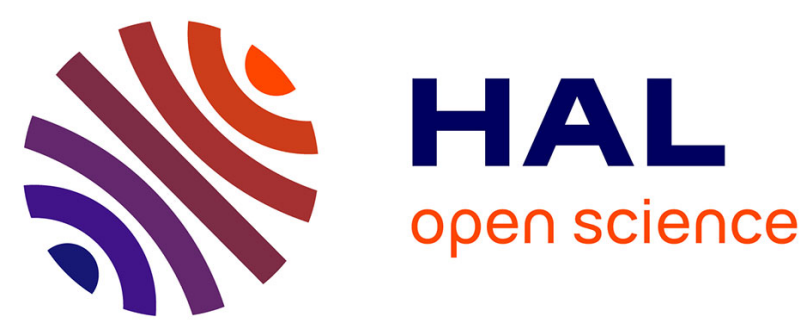

\title{
Welding Seam Classification in the Automotive Industry using Deep Learning Algorithms
}

Charbel El Hachem, Gilles Perrot, Loïc Painvin, Raphael Couturier, Jean-Baptiste Ernst-Desmulier

\section{To cite this version:}

Charbel El Hachem, Gilles Perrot, Loïc Painvin, Raphael Couturier, Jean-Baptiste Ernst-Desmulier. Welding Seam Classification in the Automotive Industry using Deep Learning Algorithms. International Conference on Industry 4.0, Artificial Intelligence, and Communications Technology (IAICT) 2021, Jul 2021, Bandung, Indonesia. hal-03549339

\section{HAL Id: hal-03549339 \\ https://hal.science/hal-03549339}

Submitted on 31 Jan 2022

HAL is a multi-disciplinary open access archive for the deposit and dissemination of scientific research documents, whether they are published or not. The documents may come from teaching and research institutions in France or abroad, or from public or private research centers.
L'archive ouverte pluridisciplinaire HAL, est destinée au dépôt et à la diffusion de documents scientifiques de niveau recherche, publiés ou non, émanant des établissements d'enseignement et de recherche français ou étrangers, des laboratoires publics ou privés. 


\section{Welding Seam Classification in the Automotive Industry using Deep Learning Algorithms}

\author{
Charbel El Hachem \\ FEMTO-ST Institute, CNRS \\ Univ. Bourgogne Franche-Comté (UBFC) \\ Belfort, France \\ charbel.el_hachem@univ-fcomte.fr
}

\author{
Raphaël Couturier \\ FEMTO-ST Institute, CNRS \\ Univ. Bourgogne Franche-Comté (UBFC) \\ Belfort, France \\ raphael.couturier@univ-fcomte.fr
}

Univ. Bourgogne Franche-Comté (UBFC) Faurecia Clean Mobility

Belfort, France

gilles.perrot@univ-fcomte.fr
Jean-Baptiste Ernst-Desmulier

$R \& D$ center

Faurecia Clean Mobility

Bavans, France

Loïc Painvin

Bavans, France

loic.painvin@faurecia.com jean-baptiste.ernst-desmulier@faurecia.com

\begin{abstract}
Welding seam inspection is key process in the automotive industry and should guarantee the quality required by the client. Visual inspection is achieved by the operator who checks each part manually, making the reliability highly improvable. That's why automating the visual inspection is needed in today's production process. Collecting data from inside the plant may not provide a balanced number of images between good welding seams and bad welding seams. In this article, we will compare a standard deep learning algorithm applied on raw data with data augmentation approaches. Our target is to reach an accuracy of $97 \%$ on the defected reference parts. This target is reached on some welds, while it remains a challenge on other welds.

Index Terms-Deep Learning, Image Classification, Convolutional Neural Network, Data Augmentation, Industry Automation.
\end{abstract}

\section{INTRODUCTION}

Welding is the process that allows to connect pieces of metal. Many industries use welding to connect parts of their products. For example, in the aviation industry [1], welding is used in manufacturing and maintenance process of the aircraft. It requires lightweight components and flawless welds to pass safety standards before taking off to the skies.

Welding is also employed in the construction of buildings, where the creation of the structural frameworks is based on metal components. Thereby, it is used to support walls, roof and floors of a building.

In the automotive industry, robustness of the welds and leakage absence are highly required. A discontinuity of a welding seam may have huge impact on the quality of the product, thereby on client's satisfaction. That is why welding seam inspection is done at each step of the assembly process.

The most fundamental reason for inspecting a weld is to determine whether its quality reaches the requirements

Supported by Faurecia for its intended application. In this paper, good welding seams will be considered as $\mathrm{OK}$ and defected welding seam will be considered as NOT OK (NOK). If the weld is NOK, rework should be applied on this weld. Acceptance criteria should be defined when measuring the two main characteristics of a specific weld: its size and the presence of discontinuities. In order to prevent weld failure due to these defects, a welding inspection is necessary and should be done right after the welding phase.

In this paper, the approach is as follows:

1) Launch an automatic solution from inside the plant that will collect data automatically.

2) Adjust dataset of real reference parts collected from the plant using data augmentation techniques.

3) Test all of the possible filters to reach high precision in detecting defective parts using MobileNet architecture.

In order to validate the automatic welding seam inspection, it should be able to reach high precision in detecting defective parts: reaching $97 \%$ of accuracy when applied on NOK parts, as requested by the client. This paper is organized as follows. Section II introduces the problem statement at the plant. Section III presents an overview of the existing work done in the area of welding seam inspection. In Section IV, the proposed supervised method for image classification is explained. In Section V, the implementation details are presented. In Section VI, the analysis of the experimental results is presented. The last section gives our concluding remarks.

\section{Problem Statement}

\section{A. Welding Seam Inspection}

Visual inspection is a necessary operation for manufacturing industries [2]-[4]. As in Figure 1, its main 


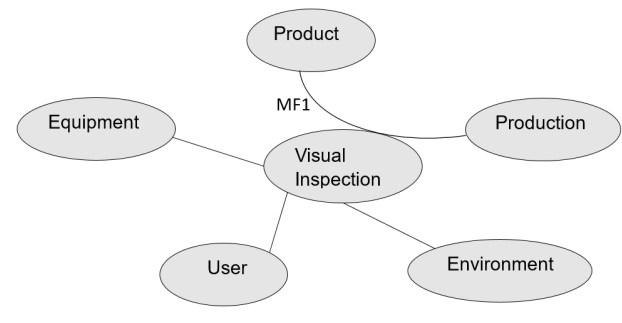

Fig. 1. Diagram representing the main function (MF1) of visual inspection and its related parameters

function (MF1) is to visually check a product during its production process. Many criteria interfere in the visual inspection process, such as:

1) Equipment: it refers to all the fixtures, technical equipment, computers and machinery used in a plant.

2) User: it is the operator who loads/unloads the product.

3) Environment: it includes all the surroundings or conditions in the plant's area (lights, dust, etc.).

These criteria's correlations may have an impact on the production line: in terms of availability, performance and quality. Visual inspection is actually done by the operator, making the reliability highly improvable [5]. The target is to automate the connection between the operator and the visual inspection. The impact of the automation on the reliability in detecting defective parts in the production line will be tracked.

\section{B. Reference Part}

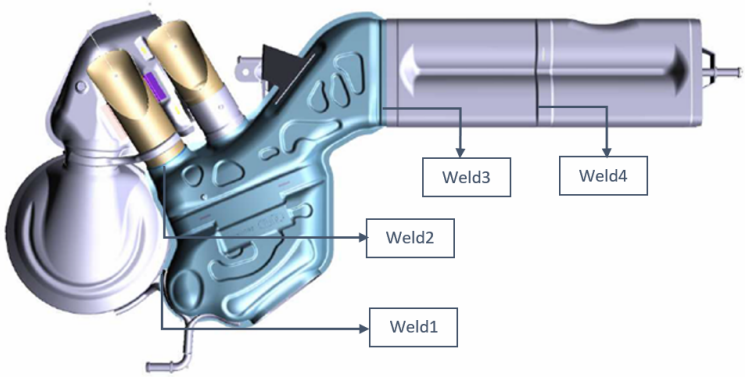

Fig. 2. Sub-assembly part to be checked

Figure 2 shows the reference part: cold line of the exhaust system, in which four welding seams need to be inspected. These weldings' type is Metal Inert Gas (MIG) and Metal Active Gas (MAG) welding: a gas metal arc welding. Figure 3 shows the reference weld $(\mathrm{OK})$ and the most frequent defects of MIG/MAG welding, as follows:

1) $\mathrm{Ok}$

2) Burn through

3) Edge not fused

4) Cold weld bead

5) Stuck wire piece

6) Interruptions

\section{Operating Procedure}

The welding seam is done by the robot. Once the welding of a specific reference part has finished, this information is sent to the Programmable Logic Controller (PLC). A PLC plays an intermediate role between the robot and the Personal Computer (PC). Its aim is to convert hexadecimal data received from the robot to binary data, understandable by the PC, and vice versa. Then, the PLC alerts the PC in order to launch the image acquisition of the reference part. This procedure is repeated each time a new reference part is welded by the robot.

After that, the leak test process takes place. It allows to ensure the proper function and maintenance of industrial systems and pipelines while calculating the loss of pressure: air diffusion in the inlet gets out from the outlet. If a pressure loss is detected, a rework should be manually applied by the operator on the concerned welding seam. After the leak test, the reference parts are stored next to each other, separated by cardboard papers. This process is called "packaging".

By verifying all welding seams, the quality of the product is guaranteed. Due to their positions, four of these welding seams are not verified by the leak test, so the operator is manually inspecting their quality. Hence, an automated visual inspection need to be implemented.
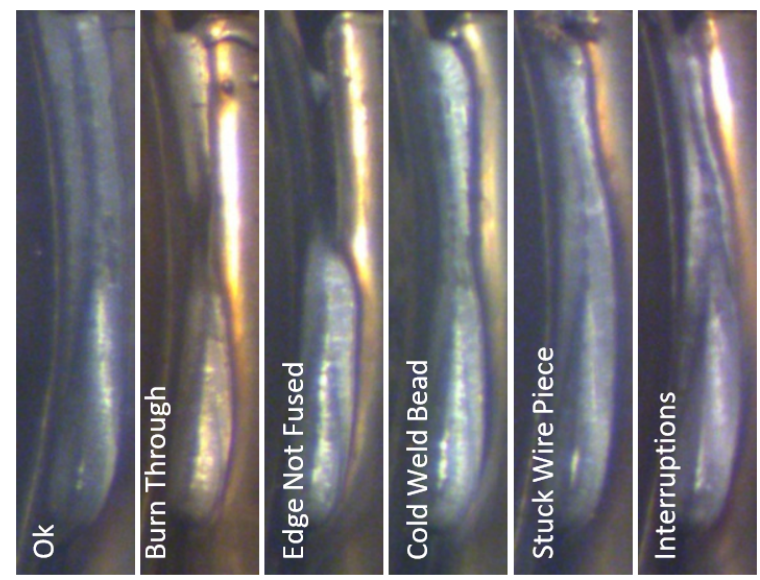

Fig. 3. Reference weld (OK) and five possible defects (NOK) for MIG/MAG welding

\section{RELATED WORKS}

Automating the classification of welding seam has been an interesting field for researches [6], [7].

Yang et al. [2] have invested in automating optical inspection of laser welding. The dataset is collected from a battery manufacturing production line. The proposed solution optimizes the Visual Geometry Group (VGG) architecture for classifying defects and reaches an accuracy of $99.87 \%$ on the test dataset.

Shevchik et al. [3] is an example of real time quality monitoring of laser welding. They applied a deep artificial neural network to reveal the unique signatures of process instabilities that can lead to defects. The confidence of the quality classification ranges between 
$71 \%$ and $99 \%$.

While Cruz et al. [4] investigated in welding inspection of liquefied petroleum gas, combining digital image processing and deep learning techniques. They proposed two inspection procedures: the first one is applied before the welding operation in order to detect the misalignment of the parts to be welded. While the second inspection procedure allows us to detect geometrical non conformities of the weld. When applying both weld inspections, the quality index was improved from $95.0 \%$ to $99.5 \%$ and has been validated in an industrial environment.

Pan et al. [8] is an example where MobileNet is applied to ensure the quality of the weld structure. They proposed a new transfer learning model based on the MobileNet architecture by adding a new Full Connection layer and a Softmax classifier. This proposed method has been tested on the welding defects dataset and the model is able to reach an accuracy of $97.69 \%$. The MobileNet architecture has proved its efficiency in image classification tasks. It will be applied on the collected dataset.

\section{Methods}

\section{A. Convolutional Neural Network}

Convolutional neural networks (CNN) has been applied on manufacturing use case and proved their adaptability to the environment's conditions [9]-[11]. In a binary classification, where the dataset is composed of two classes, a CNN will learn the specific features of a class. Once the learning phase is done, the output model will be able to predict if the given image belongs to the first or the second class. The global steps to train a neural network are as follows:

1) Data collection: images for both classes are collected; whether collected from an online dataset or live images collected from a specific use case.

2) Data cleaning: data's verification is done in this phase. Biased images should be removed. If there is any need to apply specific filters or crop before training the model, these steps should be applied in this phase.

3) Training phase: extract features from images while training the model.

4) Validation phase: at the end of each iteration, the model adjust its weights based on the data used for validation. It allows to evaluate the current state of the learning phase in terms of accuracy and loss.

In a $\mathrm{CNN}$, the input is an image following this format: $S=s \times s \times c$, where $\mathrm{S}$ is the size of the image in pixels, $s$ is the width and height of the image, and $c$ its number of channels (equal to 3 for an RGB image). Convolution phase aims to extract features from the input layer. The kernel iterates the image with all of its channels together. The kernel has a $n \times n$ size where $\mathrm{n}$ is the width and height of the kernel. As an output, the image has a size of $s 1 \times s 1 \times 1$ where $s 1=s-n+1$ and the number one represents the depth of the image.
In order to reach a depth of 256 , the defined kernel will browse the image 256 times, and the outputs will be stacked up together to create a $s 1 \times s 1 \times 256$ image. These 256 layers are called feature maps.

\section{B. MobileNet Architecture}

MobileNet Architecture proved its efficiency when applied on different domains [12]-[14], by constructing lightweight deep convolutional neural networks.

MobileNets separates the convolution phase in two smaller parts:

1) Depthwise convolution : Having the same image as input with size $S=s \times s \times c$, the same kernel size will be kept but in this time, the kernel iterates 1 channel of the image. The output is an image with size s1 where $s 1=s-n+1$ with depth $=1$.

2) Pointwise convolution: this convolution takes place after the depthwise convolution. In this case, the kernel has a size sk, where $s k=$ $1 \times 1 \times$ NumberOf InputChannel. The output of this convolution is an image with a size s1 where $s 1=s-n+1$ with depth $=1$. Then, this kernel is applied $\mathrm{f}$ times in order to obtain $\mathrm{f}$ number of feature maps. In this $\mathrm{CNN}$ example, $f=256$

When applying depthwise convolution, fewer number of parameters and fewer multiplications and additions are used. By that, the model's size and complexity are reduced. Hence, a mobileNet architecture provides smaller model to predict, which implies less execution time per prediction.

\section{Data Augmentation}

Having an unbalanced dataset makes the model's predictions biased. In Table I, the distribution of images per weld is represented, while having 5 different defects for NOK images.

TABLE I

DISTRIBUTION OF COLLECTED IMAGES FOR OK AND NOK CLASSES

\begin{tabular}{|l|l|l|l|l|}
\hline Weld area & OK training & NOK training & OK test & NOK test \\
\hline Weld1 & 100 & 100 & 1736 & 44 \\
\hline Weld2 & 50 & 50 & 1944 & 16 \\
\hline Weld3 & 90 & 90 & 1848 & 25 \\
\hline Weld4 & 150 & 150 & 1659 & 65 \\
\hline
\end{tabular}

It shows that around 2000 OK images were collected per weld, while NOK images varies between 66 and 215 per weld. The performance of a neural network is directly proportional to the amount and diversity of data available during training. Many techniques can be applied on the images in order to obtain balanced dataset [15]-[18]. One of these techniques is data augmentation, which permits better control on the given input to the neural network [17], [18]. Applying data augmentation on data can be done while training the neural network. This technique is used to increase the amount of data by adding slightly modified copies of existing data. It helps enlarging the dataset while 
providing to the neural network relevant data: data having same properties as the original dataset. By that, overfitting is reduced because the neural network treats these images as distinct images. In order to obtain better precision when predicting, filters can be applied on the images. Seven of them were tested on weld1 as shown in Figure 4. Following is the description of each applied filter:

1) RGB filter: it takes an RGB image as input and splits the 3 channels before saving each one in a separate image. It is represented in Figure 4 by 'RGB Filter Red', 'RGB Filter Green' and 'RGB Filter Blue'. This filter multiplies the original dataset by 4 .

2) Mode filter: it is used to remove noise from an image. It collects from a defined window size the most frequently occurring pixel. Then, replaces all pixels with this selected value. The kernel size used is 3, 5, 7 and 9 (represented in Figure 4 by 'Mode Filter 9'). This filter multiplies the original dataset by 5 .

3) Box blur filter: each pixel is replaced by the average of the surrounding pixels inside the defined window size (represented in Figure 4 by 'Box Blur 7'). This filter multiplies the original dataset by 5 .

4) Rank filter: Rank filters uses the local gray-level ordering to compute the filtered value (rank filter max, rank filter min and rank filter median). They are represented in Figure 4 by 'Rank Filter Max 9', 'Rank Filter Min 9', 'Rank Filter Median 9' respectively. This filter multiplies the original dataset by 5 .

5) Translation: it performs a geometric transformation which changes the position of each pixel inside the input image. 10 different variations were applied, ranging from -5 to +5 (represented in Figure 4 by 'Translation 5'). Hence, the original dataset is multiplied by 11 .

6) Rotation: a rotation of the original image is applied with an angle equal to $180^{\circ}$. It changes the sense of direction of the welding seam while keeping its geometry (represented in Figure 4 by 'Rotation 180'). This filter multiplies the original dataset by 2 .

7) Flip: this mirror effect is done by reversing the pixels. In this case, the reverse keeps the same shape of the welding seam (represented in Figure 4 by 'Flip'. This filter multiplies the original dataset by 2 .

\section{IMPLEMENTATION DETAILS}

\section{A. Data Collection}

Implementing artificial intelligence in the plants remains a challenge in today's manufacturing industries [19]-[21] Data collection was done in the plant for 8 months: collected around 250 welded reference parts per month and faced connectivity issues while transferring data outside the plant. Hence, these are

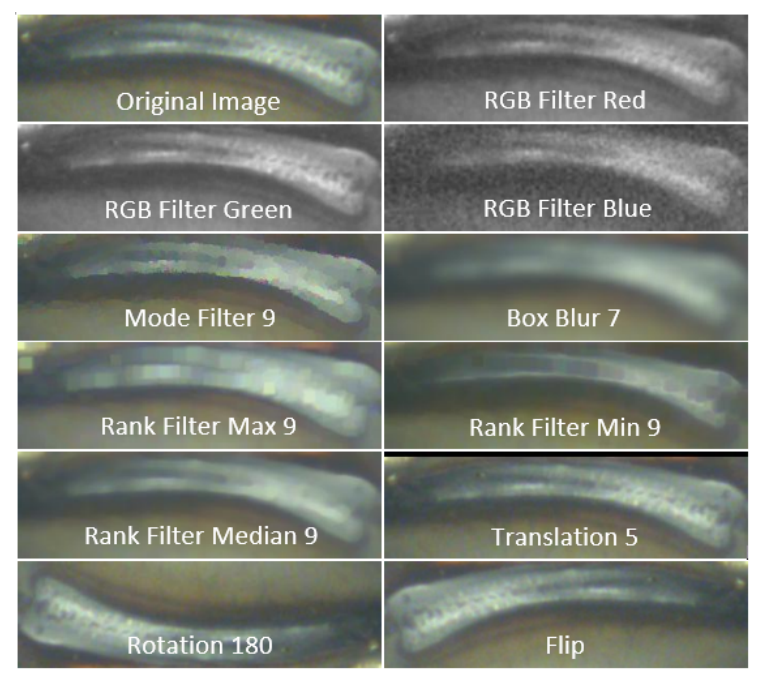

Fig. 4. Examples of data augmentation techniques applied on weld1

some of the contraints linked to the equipments' criteria as in Figure 1. The camera used is an 18MP camera, positioned at the top of the reference part with a distance of $1200 \mathrm{~mm}$ in order to cover all regions of interest. All images should be cropped so the Artificial Intelligence (AI) model can focus on the region of interest.

The crop is done prior to training to verify that the defect is still present inside the image even after the image is resized. Collected images are cropped in 4 smaller images in order to cover all of the four different welding seams. Around $80 \%$ of the collected data were used for training and $20 \%$ for testing. The classification of images is done by the plant and this classification allows the validation of the test dataset once the AI models are created.

\section{B. Analysis}

Based on the collected data, some regions of interest have less than $100 \mathrm{NOK}$ images. For example, weld2 has only 66 NOK images in total. $80 \%$ of this dataset (around 50 images) is used for training, only 16 images remains for testing. This number is not sufficient for validating the model.

Merging images from different welds may be a benefit for welds where NOK images are insufficient. This method will allow regions with low number of images (such as weld2), to benefit from regions with sufficient number of images (such as weld4). In table III, better results are obtained with the merged model (weld1-23-4) compared to a standard model (weld2).

\section{Cross Validation}

Cross validation is a resampling technique that let you ensure that your model learned your data in a correct way. In other terms, the impact of noise doesn't affect too much its decision making, guaranteeing its efficiency and accuracy [22]-[24]. So when you will show new data to your model, it will be able to predict correctly.

The method used in this case to test cross validation is 
the holdout method. The collected dataset is manually splitted in 3 sub categories: training, validation and testing. This way, the data presented as test data in the first learning phase, will be used as the training data in the second one. If the results of both trainings are close to each other, then the model got most of the patterns from the data correct.

Table II shows the results of cross-validation when applied in three different tests on weld4. Having an average of $96 \%$ and $98 \%$ on the OK and NOK predictions respectively means that the collected model is robust and will reach same accuracy once applied on live data.

TABLE II

RESULTS OF CROSS VALIDATION APPLIED ON WELD4

\begin{tabular}{|l|l|l|}
\hline Index & $\%$ OK & $\%$ NOK \\
\hline Cross validation 1 & $96 \%$ & $98 \%$ \\
\hline Cross validation 2 & $94 \%$ & $100 \%$ \\
\hline Cross validation 3 & $98 \%$ & $97 \%$ \\
\hline Average & $96 \%$ & $98 \%$ \\
\hline
\end{tabular}

\section{Experimental Environment}

The experimental environment is powered by Intel i5 CPU, 2.30 GHz with 64-bit, Windows 10 system and 8 GB memory. The software programming environment is Python. It uses Tensorflow as backend. In the image classification model, Rmsprop is selected as an optimizer of the $\mathrm{CNN}$. The learning rate decay type is exponential starting with a value of 0.01 and ending with a value equal to 0.0001 . The input of the $\mathrm{CNN}$ is a 4D array. It has a shape of (batch size, height, width, depth). The batch size (equal to 32) defines the number of samples that will be propagated through the network. The other parameters represent the height, width, and depth of the image. The input has the following values $(32,128,128,3)$. The model has been trained with 9000 epochs. Each epoch passes the entire data set through the neural network, only once.

\section{EXPERIMENTAL RESULTS}

Table III shows the results obtained on the weld1, weld2, weld 3 and weld4.

With weld1, the 'box blur' filter bypasses the target with $100 \%$ of accuracy reached on the NOK parts.

As for the weld 2 and weld3, the RGB filter provides the best results, with $93 \%$ and $96 \%$ of accuracy on NOK parts respectively.

As for weld4 the target of $97 \%$ for NOK parts is reached with several filters (mode and translation filters). The best balance of accuracy between the $\mathrm{OK}$ and the NOK parts is reached when no data augmentation is applied. This model has been chosen for live tests. The model where all welds are merged reaches the target with $98 \%$ of accuracy on NOK parts when it is trained with the mode filter.

\section{CONCLUSION}

This paper proposes a method based on deep learning and image processing to inspect welding seam. The
TABLE III

COMPARISON BETWEEN DIFFERENT DATA AUGMENTATION APPLIED ON ALL WELDING SEAMS

\begin{tabular}{|c|c|c|c|}
\hline Weld Number & Data augmentation filter & $\% \mathrm{OK}$ & $\% \mathrm{NOK}$ \\
\hline Weld1 & No data augmentation & $87 \%$ & $98 \%$ \\
\hline Weld1 & RGB & $98 \%$ & $93 \%$ \\
\hline Weld1 & Mode & $97 \%$ & $95 \%$ \\
\hline Weld1 & Box blur & $97 \%$ & $100 \%$ \\
\hline Weld1 & Rank & $\mathbf{9 8 \%}$ & $97 \%$ \\
\hline Weld1 & Translation & $97 \%$ & $98 \%$ \\
\hline Weld1 & Rotation & $98 \%$ & $93 \%$ \\
\hline Weld1 & Flip & $96 \%$ & $91 \%$ \\
\hline Weld2 & No data augmentation & $53 \%$ & $81 \%$ \\
\hline Weld2 & RGB & $70 \%$ & $93 \%$ \\
\hline Weld2 & Mode & $90 \%$ & $56 \%$ \\
\hline Weld2 & Box blur & $99 \%$ & $75 \%$ \\
\hline Weld2 & Rank & $99 \%$ & $76 \%$ \\
\hline Weld2 & Translation & $87 \%$ & $87 \%$ \\
\hline Weld2 & Rotation & $53 \%$ & $87 \%$ \\
\hline Weld2 & Flip & $15 \%$ & $100 \%$ \\
\hline Weld3 & No data augmentation & $100 \%$ & $16 \%$ \\
\hline Weld3 & RGB & $94 \%$ & $96 \%$ \\
\hline Weld3 & Mode & $100 \%$ & $36 \%$ \\
\hline Weld3 & Box blur & $98 \%$ & $72 \%$ \\
\hline Weld3 & Rank & $99 \%$ & $76 \%$ \\
\hline Weld3 & Translation & $92 \%$ & $84 \%$ \\
\hline Weld3 & Rotation & $93 \%$ & $68 \%$ \\
\hline Weld3 & Flip & $99 \%$ & $32 \%$ \\
\hline Weld4 & No data augmentation & $96 \%$ & $98 \%$ \\
\hline Weld4 & RGB & $99 \%$ & $86 \%$ \\
\hline Weld4 & Mode & $96 \%$ & $97 \%$ \\
\hline Weld4 & Box blur & $98 \%$ & $95 \%$ \\
\hline Weld4 & Rank & $98 \%$ & $93 \%$ \\
\hline Weld4 & Translation & $95 \%$ & $97 \%$ \\
\hline Weld4 & Rotation & $97 \%$ & $91 \%$ \\
\hline Weld4 & Flip & $97 \%$ & $95 \%$ \\
\hline Weld1-2-3-4 & No data augmentation & $96 \%$ & $95 \%$ \\
\hline Weld1-2-3-4 & RGB & $96 \%$ & $95 \%$ \\
\hline Weld1-2-3-4 & Mode & $97 \%$ & $95 \%$ \\
\hline Weld1-2-3-4 & Box blur & $97.5 \%$ & $95 \%$ \\
\hline Weld1-2-3-4 & Rank & $97 \%$ & $95 \%$ \\
\hline Weld1-2-3-4 & Translation & $94 \%$ & $95 \%$ \\
\hline Weld1-2-3-4 & Rotation & $91 \%$ & $96 \%$ \\
\hline Weld1-2-3-4 & Flip & $88 \%$ & $97 \%$ \\
\hline
\end{tabular}

features of the dataset are extracted by MobileNet, which fully achieves the ability to extract images' features.

The results show that the target is reached with the weld 1 and weld 4 with an accuracy of $100 \%$ and $98 \%$ respectively. The minimum accuracy is reached with the weld 2 and weld 3 with an accuracy of $93 \%$ and $96 \%$ respectively.

The target of reaching $97 \%$ of accuracy on NOK predictions is reached on weld 1 and weld 4 . The challenge remains present on weld 2 and weld 3 .

Generative Adversarial Networks (GAN) allows to obtain augmented relevant dataset. This architecture uses two neural networks: a generative model and a discriminative model, competing with each other to become more accurate in their predictions [25].

One class architecture (OC) is training the model with one class in order to extract features. Hence, One Class Convolutional Neural Network (OC-CNN): applying OC concept on a standard $\mathrm{CNN}$ reached high precision in detecting intrusions when compared to the trained cluster [26]. In our future work, we plan to apply GANs 
and OC-CNNs on the welds' dataset in order to reach the defined accuracy's target.

\section{ACKNOWLEDGMENTS}

This work was done as a part of a CIFRE ( $\mathrm{N}$ 2018/1029) project with Faurecia, funded by the Ministry of Higher Education and Research of France, managed by the Association Nationale de la Recherche et de la Technologie (ANRT) and was partially supported by the EIPHI Graduate School (contract "ANR-17-EURE0002").

The first author would like to thank Faurecia plant's staff for their availability.

\section{REFERENCES}

[1] J. Yang and M. Li, "Construction characteristics and quality control measures under high altitude and cold conditions," in IOP Conference Series: Earth and Environmental Science, vol. 676, no. 1. IOP Publishing, 2021, p. 012109.

[2] Y. Yang, L. Pan, J. Ma, R. Yang, Y. Zhu, Y. Yang, and L. Zhang, "A high-performance deep learning algorithm for the automated optical inspection of laser welding," Applied Sciences, vol. 10, no. 3, p. 933, 2020.

[3] S. Shevchik, T. Le-Quang, B. Meylan, F. V. Farahani, M. P. Olbinado, A. Rack, G. Masinelli, C. Leinenbach, and K. Wasmer, "Supervised deep learning for real-time quality monitoring of laser welding with x-ray radiographic guidance," Scientific reports, vol. 10, no. 1, pp. 1-12, 2020.

[4] Y. J. Cruz, M. Rivas, R. Quiza, G. Beruvides, and R. E. Haber, "Computer vision system for welding inspection of liquefied petroleum gas pressure vessels based on combined digital image processing and deep learning techniques," Sensors, vol. 20, no. 16, p. 4505, 2020.

[5] C. El Hachem, G. Perrot, L. Painvin, and R. Couturier, "Automation of quality control in the automotive industry using deep learning algorithms," in 2021 International Conference on Computer, Control and Robotics (ICCCR). IEEE, 2021, pp. $123-127$.

[6] X. Zhang, C. Wang, C. Xiang, C. Liu, and Y. Li, "Intelligent detection method for welding seam defects of automobile wheel hub based on yolo," in International Workshop of Advanced Manufacturing and Automation. Springer, 2020, pp. 693-702.

[7] A. Singh, V. Kalaichelvi, and R. Karthikeyan, "Application of convolutional neural network for classification and tracking of weld seam shapes for tal brabo manipulator," Materials Today: Proceedings, vol. 28, pp. 491-497, 2020.

[8] H. Pan, Z. Pang, Y. Wang, Y. Wang, and L. Chen, "A new image recognition and classification method combining transfer learning algorithm and mobilenet model for welding defects," IEEE Access, vol. 8, pp. 119951-119960, 2020.

[9] A. Kumar, G. Vashishtha, C. Gandhi, Y. Zhou, A. Glowacz, and J. Xiang, "Novel convolutional neural network (ncnn) for the diagnosis of bearing defects in rotary machinery," IEEE Transactions on Instrumentation and Measurement, vol. 70, pp. 1-10, 2021.

[10] P. Kumar and A. Shankar Hati, "Convolutional neural network with batch normalisation for fault detection in squirrel cage induction motor,' IET Electric Power Applications, vol. 15, no. 1, pp. 39-50, 2021.

[11] S. Zare and M. Ayati, "Simultaneous fault diagnosis of wind turbine using multichannel convolutional neural networks," ISA transactions, vol. 108, pp. 230-239, 2021.

[12] U. Kulkarni, S. Meena, S. V. Gurlahosur, and G. Bhogar, "Quantization friendly mobilenet (qf-mobilenet) architecture for vision based applications on embedded platforms," Neural Networks, vol. 136, pp. 28-39, 2021.

[13] R. Sadik, S. Anwar, and M. L. Reza, "Autismnet: Recognition of autism spectrum disorder from facial expressions using mobilenet architecture," International Journal, vol. 10, no. 1, 2021.

[14] J. Chen, D. Zhang, M. Suzauddola, Y. A. Nanehkaran, and Y. Sun, "Identification of plant disease images via a squeezeand-excitation mobilenet model and twice transfer learning," IET Image Processing, vol. 15, no. 5, pp. 1115-1127, 2021.
[15] S. Kotsiantis, D. Kanellopoulos, P. Pintelas et al., "Handling imbalanced datasets: A review," GESTS International Transactions on Computer Science and Engineering, vol. 30, no. 1, pp. 25-36, 2006.

[16] L. Mena and J. A. Gonzalez, "Symbolic one-class learning from imbalanced datasets: application in medical diagnosis," International Journal on Artificial Intelligence Tools, vol. 18, no. 02, pp. 273-309, 2009.

[17] F. Zhou, S. Yang, H. Fujita, D. Chen, and C. Wen, "Deep learning fault diagnosis method based on global optimization gan for unbalanced data," Knowledge-Based Systems, vol. 187, p. 104837, 2020.

[18] A. Mikołajczyk and M. Grochowski, "Data augmentation for improving deep learning in image classification problem," in 2018 international interdisciplinary PhD workshop (IIPhDW). IEEE, 2018, pp. 117-122.

[19] J. Marcus, "Challenges and frontiers in implementing artificial intelligence in process industry," Impact and Opportunities of Artificial Intelligence Techniques in the Steel Industry: Ongoing Applications, Perspectives and Future Trends, vol. 1338, p. 1, 2021.

[20] G. Goh, S. Sing, and W. Yeong, "A review on machine learning in 3d printing: Applications, potential, and challenges," Artificial Intelligence Review, vol. 54, no. 1, pp. 63-94, 2021.

[21] B. Ralph and M. Stockinger, "Digitalization and digital transformation in metal forming: key technologies, challenges and current developments of industry 4.0 applications," in Proceedings of the XXXIX. Colloquium on Metal Forming, 2021.

[22] M. J. Abdulaal, A. J. Casson, and P. Gaydecki, "Critical analysis of cross-validation methods and their impact on neural networks performance inflation in electroencephalography analysis," IEEE Canadian Journal of Electrical and Computer Engineering, vol. 44, no. 1, pp. 75-82, 2021.

[23] A. Steland and B. E. Pieters, "Cross-validation and uncertainty determination for randomized neural networks with applications to mobile sensors," arXiv preprint arXiv:2101.01990, 2021.

[24] A. Oskooei, S. M. Chau, J. Weiss, A. Sridhar, M. R. Martínez, and B. Michel, "Destress: deep learning for unsupervised identification of mental stress in firefighters from heart-rate variability (hrv) data," in Explainable AI in Healthcare and Medicine. Springer, 2021, pp. 93-105.

[25] I. J. Goodfellow, J. Pouget-Abadie, M. Mirza, B. Xu, D. WardeFarley, S. Ozair, A. Courville, and Y. Bengio, "Generative adversarial networks," arXiv preprint arXiv:1406.2661, 2014.

[26] P. Oza and V. M. Patel, "One-class convolutional neural network," IEEE Signal Processing Letters, vol. 26, no. 2, pp. 277281, 2018. 